[Article]

www.whxb.pku.edu.cn

\title{
芴-含氮芳杂环共聚物的载流子注入特性
}

\author{
孙冠楠 $^{1}$ 许 海 $^{1,2}$ 刘晓冬 ${ }^{2} \quad$ 刘 丹丹 $^{1}$ \\ 杨 兵 $1,2, *$ 田雷蕾 1 马於光 ${ }^{1, *}$ \\ ('吉林大学超分子结构与材料国家重点实验室, 长春 130012；2吉林大学化学学院, 长春 130012)
}

\begin{abstract}
摘要：为了改善聚芴的载流子注人特性, 采用密度泛函理论 B3LYP/6-31G ${ }^{*}$ 方法计算比较了芴、芴-联吡啶和 芴-菲咯啉低聚物的几何结构、电子结构、最低激发能及重组能等, 并外推到相应聚合物. 结果发现: 联吡啶/菲咯 啉含氮芳杂环的缺电子性质能够诱导聚芴的最高占据轨道(HOMO) 和最低空轨道 (LUMO)能级分别下降 $0.45 /$ $0.47 \mathrm{eV}$ 和 $0.32 / 0.38 \mathrm{eV}$, 提高电子注人能力的同时, 调控载流子注人平衡; 联吡啶单元的引入导致电子和空穴重 组能升高(降低聚芴的载流子迁移率), 而芴-菲咯啉共聚物显示了与聚芴相似的迁移性能.
\end{abstract}

关键词： 聚芴; 联吡啶; 菲咯啉; 载流子注人; 量子化学

中图分类号： 0641

\section{Carrier Injection Property of Fluorene-Aromatic Nitrogen Heterocycle Copolymers}

\author{
SUN Guan-Nan ${ }^{1} \quad$ XU Hai ${ }^{1,2} \quad$ LIU Xiao-Dong ${ }^{2} \quad$ LIU Dan-Dan ${ }^{1}$ \\ YANG Bing ${ }^{1,2, *} \quad$ TIAN Lei-Lei ${ }^{1} \quad$ MA Yu-Guang ${ }^{1, *}$ \\ ('State Key Laboratory of Supramolecular Structure and Materials, Jilin University, Changchun 130012, P. R. China; \\ ${ }^{2}$ College of Chemistry, Jilin University, Changchun 130012, P. R. China)
}

\begin{abstract}
To improve the carrier-injection of polyfluorene, oligomers of polyfluorene, fluorene-bipyridine, and fluorene-phenanthrolin copolymers were theoretically studied for their geometries, electronic structures, excitation energies, and reorganization energies by density functional theory at the B3LYP/6-31G level. We evaluated the properties of the corresponding polymers by linear extrapolation. We found a large decrease of about $0.45 / 0.47 \mathrm{eV}$ and $0.32 / 0.38 \mathrm{eV}$ for the highest occupied molecular orbital (HOMO) and the lowest unoccupied molecular orbital (LUMO) levels, respectively, after the introduction of electron-deficient bipyridine/phenanthrolin into polyfluorene. These molecules improved the electron injection ability and the balance of carrier-injection in polyfluorene systems. Additionally, the reorganization energy increased for both electron and hole transports upon the incorporation of bipyridine, which resulted in worse carrier mobility. However, the fluorene-phenanthrolin copolymer showed a similar charge transport property to polyfluorene.
\end{abstract}

Key Words: Polyfluorene; Bipyridine; Phenanthrolin; Carrier-injection; Quantum chemistry

$\pi$ 共轭聚合物因良好的成膜加工性能、易于调 节的能带结构等优点, 广泛用于有机发光二极管、太 阳能电池、薄膜晶体管及传感器等方面的研究 ${ }^{[1-6]}$. 聚
芴 $(\mathrm{PF})$ 是一类高效率的蓝色电致苂光高分子材料, 一 直深受研究者的关注. 但是, 聚芴由于阴极的空穴注 人势垒远比阳极的电子注人势垒高 ${ }^{[7-9]}$ (如图 1 所示),

Received: March 18, 2010; Revised: May 14, 2010; Published on Web: June 29, 2010.

*Corresponding authors. Email: ygma@mail.jlu.edu.cn, yangbing@mail.jlu.edu.cn; Tel: +86-431-85168492.

The project was supported by the National Key Basic Research Program of China (973) (2009CB623605) and National Natural Science Foundation of China (20834006, 20774034).

国家重点基础研究发展规划项目(973) (2009CB623605)和国家自然科学基金(20834006, 20774034)资助

(C) Editorial office of Acta Physico-Chimica Sinica 


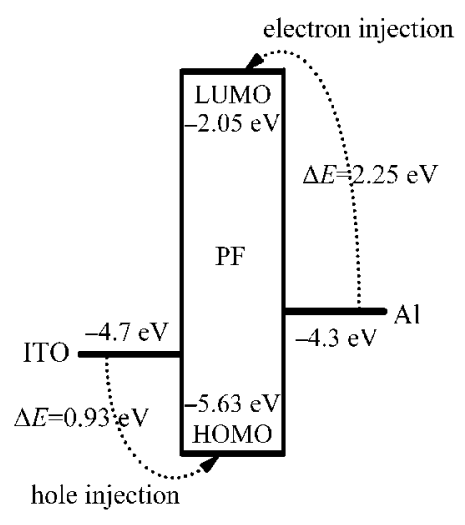

图 1 聚芴(PF)空穴和电子注入势垒

Fig.1 Energy barriers of hole and electron injections into polyfluorene $(\mathrm{PF})$ ITO: indium tin oxide

有机聚合物发光层大多以空穴为多数载流子. 因此, 发光层中电子和空穴严重不平衡, 造成发光通常发 生在靠近阴极附近, 直接导致电致发光效率降低. 我 们课题组 ${ }^{[10-15]}$ 曾把联吡啶和菲咯啉作为配体单元引 人聚芴主链,研究了其对不同金属离子的苂光传 感, 而本文拟利用此类含氮芳杂环的缺电子性质, 来提高电子的注人能力, 获得 $\mathrm{N}$ 型共轭聚合物材料, 并对其迁移性能进行简单评价.

\section{1 理论方法}

运用 Gaussian 03 量子化学程序 ${ }^{[16]}$, 采用密度泛 函理论 B3LYP 方法 ${ }^{[17-18]}$, 在 6-31G* 基组水平上依次 对芴((flu-flu $)_{n}, n=1-4$, 下同)、芴-联吡啶((flu-bpy $)_{n}$ ) 和芴-菲咯啉低聚物((flu-phe) $\left.)_{n}\right)$ 的几何结构进行全 优化. 在优化的基态几何构型的基础上, 用中间忽 略微分重叠(ZINDO)、含时密度泛函理论(TD-DFT) 方法计算最低激发能 ${ }^{[19]}$, 以低聚物重复单元数目 $n$ 的倒数 $(1 / n)$ 与性质的线性关系外推聚合物 $(n=\infty)$ 的 性质 ${ }^{[20-22]}$.

高温条件下, 有机材料中电荷迁移以跳跃机制 为主导, 类似于非绝热的电子转移反应, 可以用 Marcus 理论 ${ }^{[23-24]}$ 来描述电荷在相邻分子间的跳跃速 率 $k_{\mathrm{et}}$ :

$$
k_{\mathrm{el}}=\left(4 \pi^{2} / h\right) H_{\mathrm{AB}}^{2}\left(4 \pi \lambda k_{\mathrm{B}} T\right)^{-1 / 2} \exp \left(-\lambda / 4 k_{\mathrm{B}} T\right)
$$

其中 $h$ 是普朗克常数; $k_{\mathrm{B}}$ 是波尔兹曼常数; $T$ 是热力 学温度; $H_{\mathrm{AB}}$ 是电子耦合矩阵元, 表示相邻给-受体分 子之间的电子耦合作用程度; $\lambda$ 是重组能, 包括内重 组能和外重组能. 内重组能是由于电子转移过程中, 分子结构弛豫而引起的能量变化; 外重组能来源于
环境分子的极化效应, 固体中基本可以忽略. 无定型 薄膜中, 由于分子结构和平均堆积结构的相似性, 可 以近似认为同系列聚合物分子间的 $H_{\mathrm{AB}}$ 变化不大, 这样分子内重组能成为评估材料迁移性质的重要因 素. 分子内重组能的计算过程有很多文献报道 ${ }^{[25-30]}$, 内重组能计算公式为: $\lambda_{\text {in }}=\lambda_{1}+\lambda_{2}$, 其中, $\lambda_{1}$ 是离子态 在中性几何构型和平衡几何构型时的能量差, $\lambda_{2}$ 是 中性态在离子几何构型与平衡几何构型时的能量 差. 这四个能量用 B3LYP/6-31G* 方法基组 ${ }^{[30]}$ 计算得 到.

\section{2 结果与讨论}

\section{1 几何构型优化}

通过对芴((flu-flu) $\left.)_{n}\right)$ 、芴-联吡啶((flu-bpy) $\left.)_{n}\right)$ 、芴菲咯啉((flu-phe) $)_{n}$ )低聚物(图 2)进行结构优化. 发现 随重复单元数 $n(n=1-4)$ 增大, 低聚物各自中间链段 的键长、键角、二面角变化趋于稳定. 与 (flu-flu) ${ }_{n}$ 相 比, (flu-bpy) ${ }_{n}$ 和 (flu-phe) ${ }_{n}$ 中芴单元的键长、键角变 化不明显. 在各重复单元内部, 连接芴单元、荡与联 吡啶、芴与菲咯啉的单键键长顺序为: (flu-flu) ${ }_{n}>$ (flubpy $)_{n} \approx(\text { flu-phe })_{n}$; 芴与芴、芴与联吡啶、荡与菲咯啉 之间所成的二面角相近, 都在 $37^{\circ}$ 左右; 芴单元和菲 咯啉单元分别是平面结构, 而联吡啶中两个吡啶环 之间成约 $32^{\circ}$ 的二面角.

\section{2 前线分子轨道能级}

如图 3 所示, 分析了(flu-flu) ${ }_{n}$ (flu-bpy) ${ }_{n}$ 和(flu-phe) ${ }_{n}$ $(n=1)$ 的 HOMO 和LUMO 的电子云分布. 对于 (fluflu $)_{n}$, HOMO 和 LUMO 均离域在整个分子链上. 而 在(flu-bpy $)_{n}$ 和(flu-phe) ${ }_{n}$ 中, HOMO 和 LUMO 的电子
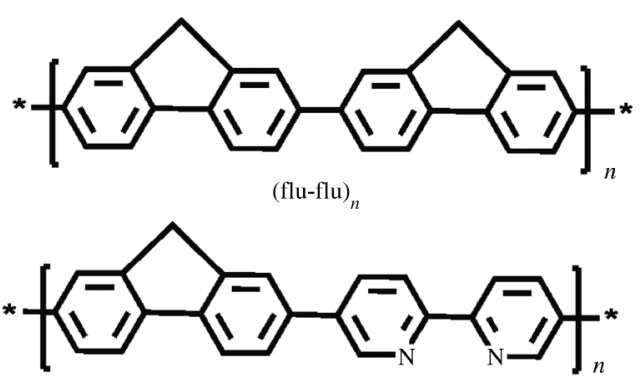

(flu-bpy)

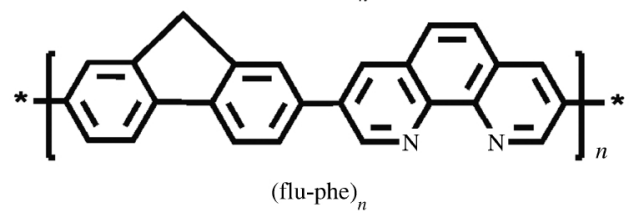

图 2 低聚物分子结构

Fig.2 Molecular structures of oligomers 


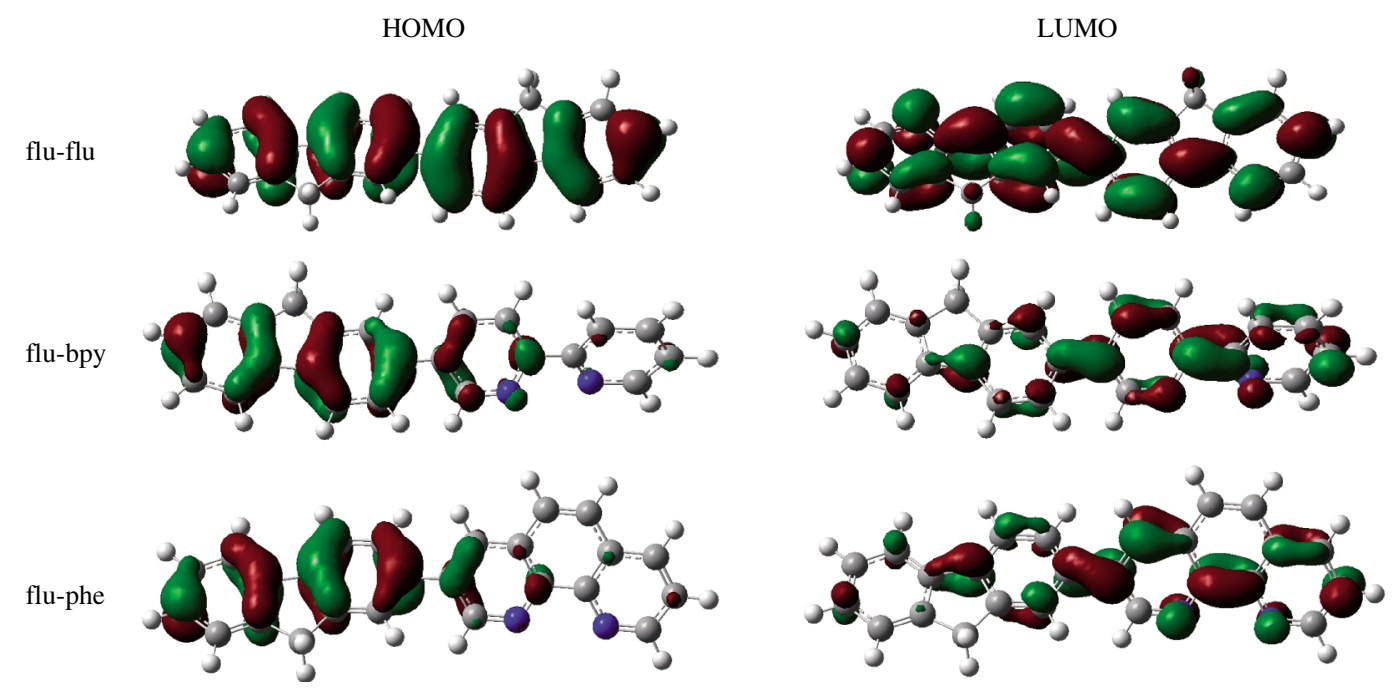

图 3 低聚芴及其衍生物的前线分子轨道图

Fig.3 HOMO and LUMO of oligomers of fluorene and its derivatives

云分布相似, 均发生相似程度的局域分离. HOMO 主要局域在芴及与芴相连的吡啶环上, LUMO 主要 局域在联吡啶和菲咯啉单元以及与之相连的苯环 上. 以上电子云分布特征主要是由含氮芳杂环(联吡 啶/菲咯啉)的缺电子性质所决定的 ${ }^{[31-32]}$.

图 4 中显示了低聚芴及其衍生物的能级分布. 从图 4 可以看出前线分子轨道能级随重复单元数 $n$ 增大的变化情况. 值得注意的是, 联吡啶和菲咯啉单 元的引人, (flu-bpy) ${ }_{n}$ 和(flu-phe) ${ }_{n}$ 的 HOMO 和LUMO 能级发生整体下移, 有利于增强电子的注人能力, 促 进载流子注人平衡. 随重复单元数 $n$ 增加, $\mathrm{HOMO}$ 和 LUMO 能级的变化幅度都呈逐渐减小趋势(逐渐 接近聚合物的有效共轭长度). 进一步提高分子链内
联吡定单元与芴单元的比例 (3:1), (flu-3bpy) $)_{n / 2}$ 的分 子结构式如图 4 所示. 考虑到分子结构相近才具有 可比较性, 故我们选取(flu-bpy $)_{n}(n=2 、 4 、 6)$ 的低聚物 与 (flu-3bpy $)_{n / 2}(n=2 、 4 、 6)$ 进行比较, 后面涉及(flu$3 \mathrm{bpy})_{n / 2}$ 的比较均采取相同处理方法. 与 (flu-bpy) ${ }_{n}$ 相比, (flu-3bpy $)_{n / 2}$ 的 HOMO 、LUMO 能级分别具有 进一步下降的趋势.

对 HOMO、LUMO 能级与重复单元的倒数 $1 / n$ 进行线性拟合, 并外推到 $n=\infty$ 时得到聚合物的相 应性质(图 5). 可以看到, 联吡啶和菲咯啉单元的引 人, 分别诱导聚芴的 LUMO 能级下降了 0.32 和 $0.38 \mathrm{eV} ; \mathrm{HOMO}$ 能级下降了 0.45 和 $0.47 \mathrm{eV}$. 一方 面, 说明含氮芳杂环的引人, 可以同时调节HOMO、

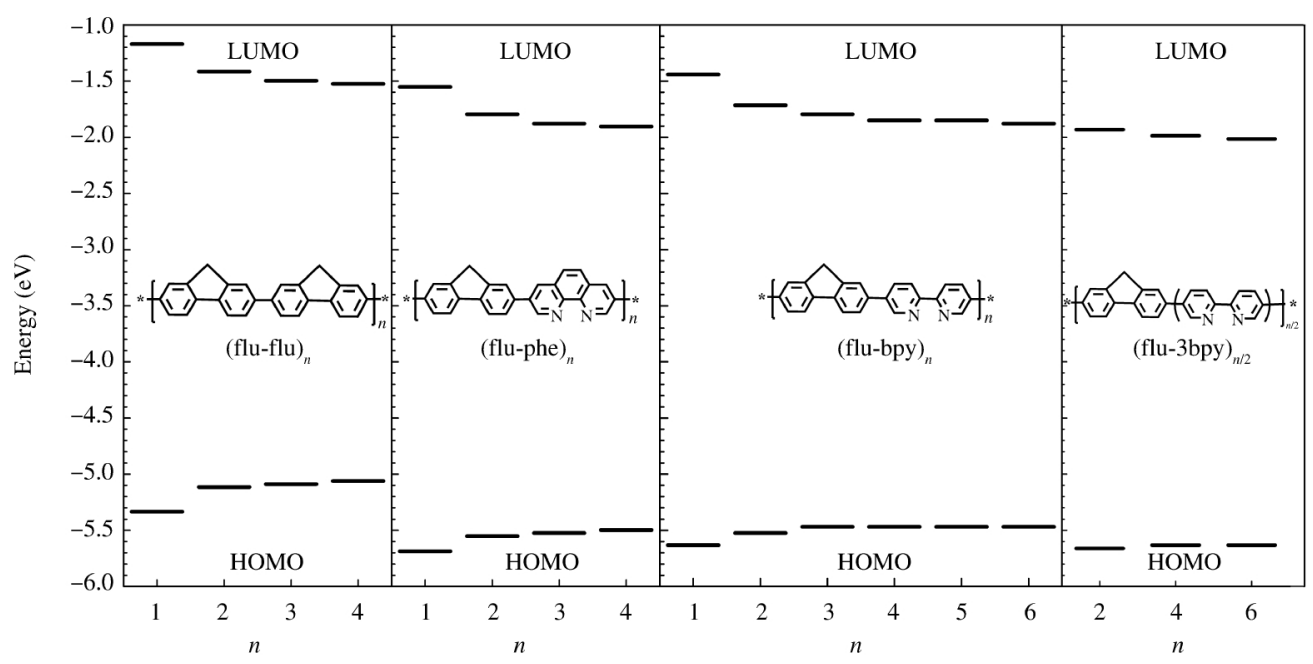

图 4 低聚芴及其衍生物的能级分布

Fig.4 Energy level diagrams of oligomers of fluorene and its derivatives 

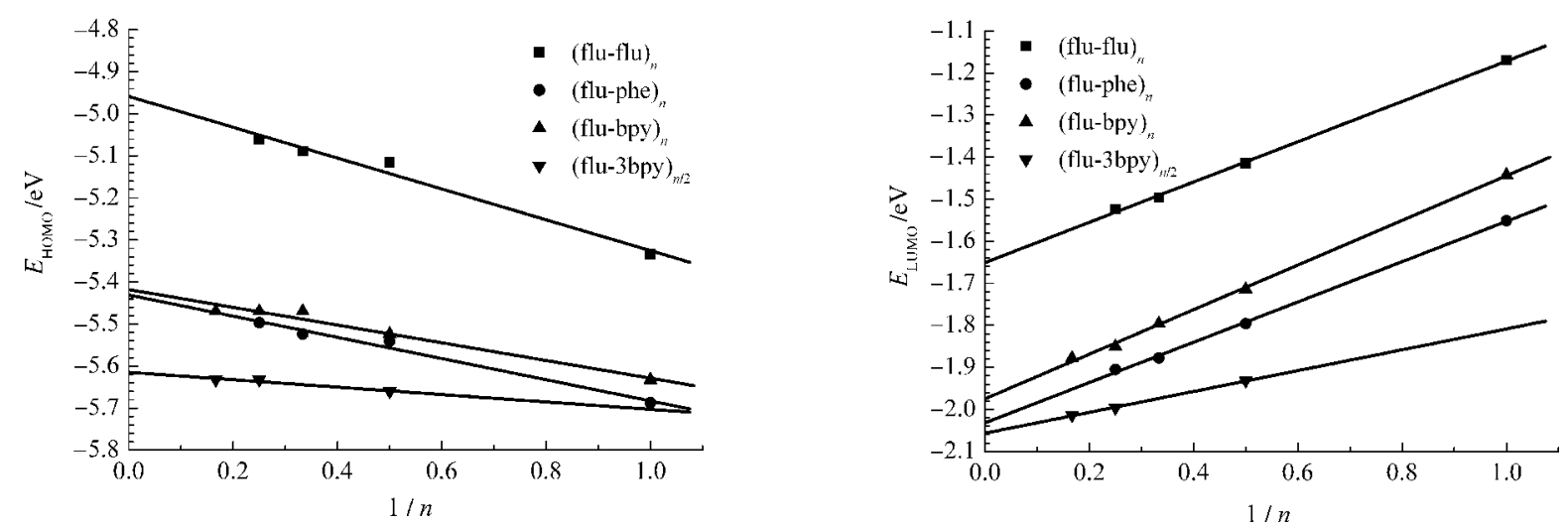

图 5 低聚芴及其衍生物的 HOMO、LUMO 能级的外推 $(n=\infty)$

Fig.5 HOMO, LUMO level extrapolation $(n=\infty)$ on oligomers of fluorene and its derivatives

LUMO 能级的整体下降, 既增强了电子的注人能力, 同时又调控了空穴与电子的注人平衡, 从图 1 可以 应证; 另一方面, 芴-菲咯啉共聚物 HOMO 、LUMO 能级的下降程度更大, 说明平面大 $\pi$ 共轭的菲咯啉 单元比扭曲结构的联吡啶单元具有更强的缺电子特 征. 此外, 进一步提高分子链内联吡啶单元的比例, 与(flu-bpy) ${ }_{n}$ 相比, (flu-3bpy) $)_{n / 2}$ 的HOMO 、LUMO 能级 分别进一步下降 0.20 和 $0.08 \mathrm{eV}$.

\subsection{HOMO-LUMO 能隙和最低激发能}

HOMO-LUMO 能隙是电子激发过程所需要的 最低能量, 它是反映物质发光性质的一个重要参数 ${ }^{[21]}$.

表 1 中列出了低聚芴及其衍生物的 HOMOLUMO 能隙及最低激发能. 如表 1 所示, 总体上, 低 聚苆及其衍生物随重复单元数 $n$ 的增加, 主链的共 轭程度增大, HOMO-LUMO 能隙和最低激发能均呈 递减、收玫趋势, 逐渐接近聚合物的有效共轭长度. 利用低聚物重复单元的倒数 $1 / n$ 与 HOMO-LUMO
能隙和最低激发能之间的线性关系外推到 $n=\infty$ 时 聚合物的性质. 我们发现, 聚芴及其衍生物 $(n=\infty)$ 在HOMO-LUMO 能隙 $\left(\Delta E_{\text {LUMO-HOMO }}\right)$ 和最低激发能 $\left(E_{\mathrm{g}(\mathrm{ZNDO}) 、} E_{\mathrm{g}(\mathrm{TD}-\mathrm{DFT})}\right)$ 指标上的变化趋势基本一致, 为 (flu-flu $)_{n}<$ (flu-bpy $)_{n} \approx$ (flu-phe $)_{n}$; 从结果上看, ZINDO 方法的结果更接近实验值 ${ }^{[33}$, 这可能是由于 ZINDO 方法使用的经验参数更适合此研究体系 ${ }^{[34]}$, 而 TDDFT 方法系统地低估了激发能约 0.3-0.4 $\mathrm{eV}^{[35-36]}$. 与 (flu-flu) ${ }_{n}$ 相比, (flu-bpy) ${ }_{n}$ 和(flu-phe) ${ }_{n}$ 增大的 HOMOLUMO能隙和最低激发能会导致共聚物发光波长 轻微蓝移, 使其保持蓝色发光, 与实验结果 ${ }^{[9-10]}$ 基本 一致. (flu-bpy $)_{n}$ 和(flu-phe) ${ }_{n}$ 的 HOMO-LUMO 能隙 增宽, 主要归功于含氮芳杂环(联吡啶/菲咯啉)的诱 导 HOMO 能级更大幅度的下降. 另一方面, 含氮芳 杂环(联吡啶/菲咯啉)的引人, 造成(flu-bpy) ${ }_{n}$ 和(fluphe $)_{n}$ 的 HOMO/LUMO 电子云密度发生局域分离, 相当于增大了电子在共聚物主链上离域的势垒, 降

表 1 低聚芴及其衍生物的 HOMO-LUMO 能隙 $\left(\Delta \boldsymbol{E}_{\text {LUмо-номо }}\right)$ 及最低激发能 $\left(\boldsymbol{E}_{\mathrm{g}}\right)$

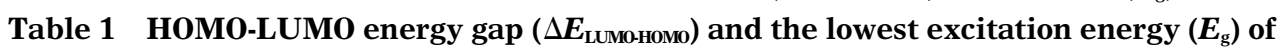
the oligomers of fluorene and its derivatives

\begin{tabular}{|c|c|c|c|c|c|c|c|c|}
\hline & \multirow{2}{*}{ Oligomer } & \multicolumn{6}{|c|}{$n$} & \multirow{2}{*}{ Expt. } \\
\hline & & 1 & 2 & 3 & 4 & 6 & $\infty$ & \\
\hline \multirow[t]{3}{*}{$\Delta E_{\text {шимо-номо }} / \mathrm{eV}$} & (flu-flu $)_{n}$ & 4.16 & 3.70 & 3.59 & 3.53 & & 3.31 & \\
\hline & (flu-phe) ${ }_{n}$ & 4.14 & 3.76 & 3.65 & 3.59 & & 3.40 & \\
\hline & $(\text { flu-3bpy })_{n / 2}$ & & 3.78 & & 3.65 & 3.62 & 3.50 & \\
\hline \multirow{2}{*}{$E_{\mathrm{g}(\mathrm{ZINDO})} / \mathrm{eV}$} & (flu-phe) ${ }_{n}$ & 3.64 & 3.49 & 3.42 & 3.39 & & 3.30 & $3.35^{[10]}$ \\
\hline & $(\text { flu-3bpy })_{n / 2}$ & & 3.57 & & 3.44 & 3.42 & 3.33 & \\
\hline \multirow[t]{2}{*}{$E_{\mathrm{g}(\mathrm{TD}-\mathrm{DFT})} / \mathrm{eV}$} & $(\text { flu-flu })_{n}$ & 3.80 & 3.30 & 3.17 & 3.11 & & 2.88 & \\
\hline & $(\text { flu-bpy })_{n}$ & 3.81 & 3.37 & 3.25 & 3.20 & 3.16 & 2.98 & \\
\hline
\end{tabular}


低其 $\pi$ 共轭程度, 导致 HOMO-LUMO 能隙和最低 激发能增大. 另外, 扭曲的联吡啶单元和平面的菲咯 啉单元并没有明显影响(flu-bpy) ${ }_{n}$ 和(flu-phe) ${ }_{n}$ 的能隙 和最低激发能. 但增加分子链内联吡啶单元的比例, 与(flu-bpy $)_{n}$ 相比, (flu-3bpy $)_{n / 2}$ 的能隙和最低激发能 呈轻微增加趋势.

\section{4 重组能}

为了研究以上聚芴及其衍生物的载流子迁移性 质, 分别计算了其低聚物的电子和空穴重组能 $\left(\lambda_{\mathrm{e}}\right.$ 和 $\lambda_{\mathrm{h}}$ ). 因为对于无定型聚合物体系, 重组能是决定载 流子迁移率的一个重要因素. 根据 Marcus 电子转移 理论 ${ }^{[23-24]}$, 重组能越小, 迁移率性能越高. 如图 6 所 示, 当 $n=1$ 时, 聚合物的重组能明显偏离了线性关 系, 故只选取 $n=2-4$ 的结果进行拟合 ${ }^{[37]}$. 总体上, 这 四种聚合物均呈现电子重组能高于空穴重组能的趋 势, 反映为空穴传输能力强于电子传输能力; 同时, 随着 $n$ 增大, 聚合物的电子和空穴重组能均呈降低 趋势, 表示聚合物链的刚性随链长扩展而逐渐增强, 聚合物相对于低聚物有更高的迁移率. 与(flu-flu) ${ }_{n}$ 相比, (flu-phe) ${ }_{n}$ 具有与之非常相似的电子和空穴重
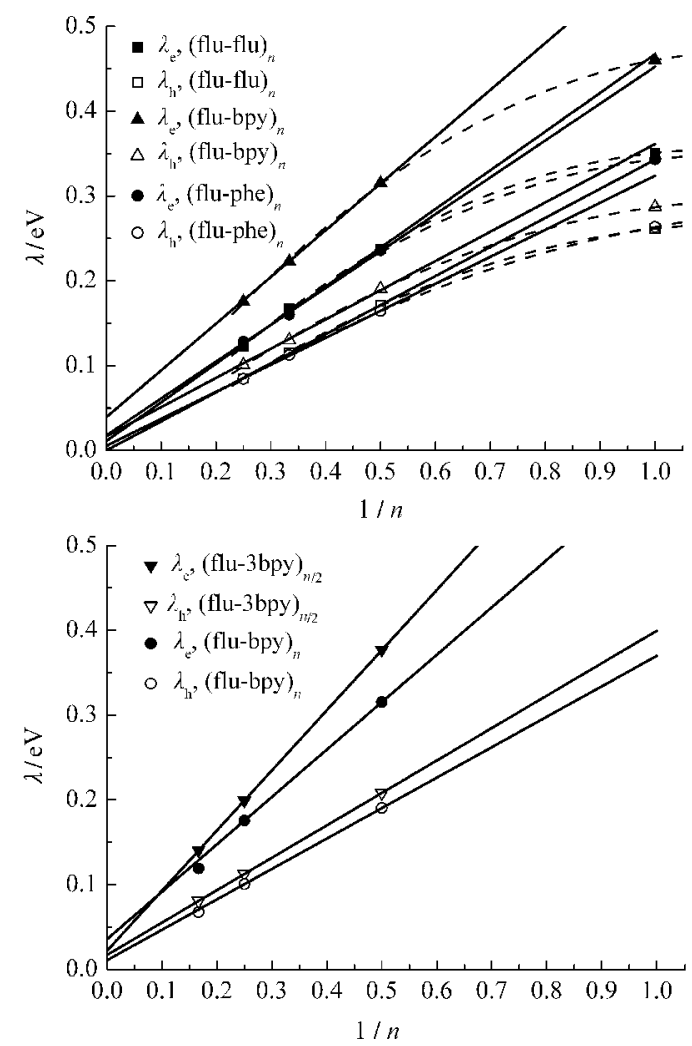

图 6 低聚芴及其衍生物的电子 $\left(\lambda_{\mathrm{e}}\right)$ 和空穴 $\left(\lambda_{\mathrm{h}}\right)$ 重组能

Fig.6 Electron $\left(\lambda_{\mathrm{e}}\right)$ and hole $\left(\lambda_{\mathrm{h}}\right)$ reorganization energies of the oligomers of fluorene and its derivatives
组能, 而(flu-bpy) ${ }_{n}$ 的电子和空穴重组能均较(flu-flu) 提高. 这可由组成聚合物链的芴单元和菲咯啉单元 具有相似的刚性来解释, 相反, 联吡定单元中可旋转 的吡啶环降低了聚合物链的刚性, 是电子和空穴重 组能升高的主要根源. 因此, 联吡啶单元的引人, 理 论上会降低聚芴的电子和空穴整体的迁移率. 值得 注意的是, 当进一步提高分子链内联吡啶单元比例 时, 虽然与(flu-bpy) ${ }_{n}$ 相比, (flu-3bpy) ${ }_{n / 2}$ 的电子和空 穴重组能进一步增大, 但是我们发现, 当 $n=\infty$ 时 $(\text { flu- } 3 b p y)_{n / 2}$ 具有更为相近的电子和空穴重组能, 意 味着通过控制联吡啶单元的比例在一定程度上可以 获得平衡的电子和空穴迁移性能. 这是因为联吡啶 单元数目的增加提高了 LUMO 局域部位的刚性所 致, 相当于减小了电子重组能.

\section{3 结 论}

通过把具有缺电子性质的联吡啶或菲咯啉单元 引人聚芴, 诱导聚芴前线轨道能级的整体下降, 其中 LUMO 能级分别下降了 0.32 和 $0.38 \mathrm{eV} ; \mathrm{HOMO}$ 能 级下降了 0.45 和 $0.47 \mathrm{eV}$, 有利于增强电子的注人 能力, 促进载流子注人平衡. 外推得到聚䓄和芴-联 吡啶、荡-菲咯啉共聚物的 HOMO-LUMO 能隙和最

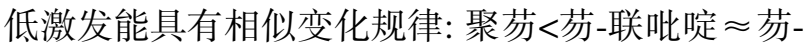
菲咯啉. 与聚芴相比, 芴-联吡啶和芴-菲咯啉增大的 HOMO-LUMO 能隙和最低激发能有利于保证共聚 物的蓝光发射, 而芴-联吡啶和芴-菲咯啉共聚物之 间无明显差异. 聚苆和芴-菲咯啉共聚物具有非常相 似的电子和空穴重组能, 而芴-联吡啶共聚物的电子 和空穴重组能均高于聚芴, 这取决于联吡啶和菲咯 啉单元的刚性程度.

\section{References}

1 Kraft, A.; Grimsdale, A. C.; Holmes, A. B. Angew. Chem. Int. Edit., 1998, 37: 402

2 Mitschke, U.; Bäuerle, P. J. Mater. Chem., 2000, 10: 1471

3 Brabec, C. J.; Sariciftci, N. S.; Hummelen, J. C. Adv. Funct. Mater., 2001, 11: 15

4 Horowitz, G. Adv. Mater., 1998, 10: 365

5 McQuade, D. T.; Pullen, A. E.; Swager, T. M. Chem. Rev., 2000, 100: 2537

6 Hou, J. H.; Tan, Z. A.; Yan, Y.; He, Y. J.; Yang, C. H.; Li, Y. F. J. Am. Chem. Soc., 2006, 128: 4911

7 Braun, D.; Heeger, A. J. Appl. Phys. Lett., 1991, 58: 1982

8 Yamamoto, T.; Anzai, K.; Iijima, T.; Fukumoto, H. Macromolecules, 2004, 37: 3064 
9 Pei, Q. B.; Yang, Y. J. Am. Chem. Soc., 1996, 118: 7416

10 Zhang, M.; Lu, P.; Ma, Y. G.; Shen, J. C. J. Phys. Chem. B, 2003, 107: 6535

11 Zhang, M.; Lu, P.; Tian, L. L.; Zhang, W.; Yang, B.; Ma, Y. G. Feng, J. K. Acta Phys. -Chim. Sin., 2004, 20: 924 [张 明, 路 萍, 田雷蕾, 张 武, 杨 兵, 马於光, 封继康. 物理化学 学报, 2004, 20: 924]

12 Zhang, M.; Lu, P.; Wang, X. M.; He, L.; Xia, H.; Zhang, W.; Yang, B.; Liu, L. L.; Yang, L.; Yang, M.; Ma, Y. G.; Feng, J. K.; Wang, D. J.; Tamai, N. J. Phys. Chem. B, 2004, 108: 13185

13 Yang, B.; Tian, L. L.; Zhang, H. Y.; Zhang, W.; Liu, X. D.; Xu, H.; Xie, Z. Q.; Lu, P.; Zhang, M.; Yu, J. S.; Ma, Y. G.; Shen, J. C. J. Phys. Chem. B, 2006, 110: 16846

14 Zhang, W.; Yang, B.; Lu, P.; Li, M.; Tian, L. L.; Ma, Y. G. Synth. Met., 2008, 158(5): 194

15 Tian, L. L.; Zhang, W.; Yang, B.; Lu, P.; Zhang, M.; Lu, D.; Ma, Y. G.; Shen, J. C. J. Phys. Chem. B, 2005, 109: 6944

16 Frisch, M. J.; Trucks, G. W.; Schlegel, H. B.; et al. Gaussian 03. Revision B.03. Pittsburgh, PA: Gaussian Inc., 2003

17 Lee, C.; Yang, W.; Parr, R. G. Phys. Rev. B, 1988, 37: 785

18 Becke, A. D. J. Chem. Phys., 1993, 98: 5648

19 Xu, H.; He, F.; Yang, B.; Zhang, H. Y.; Liu, S. J.; Gu, X.; Liu, D. D.; Liu, X. D.; Yu, J. S.; Ma, Y. G. Acta Phys. -Chim. Sin., 2009, 25: 869 [许 海, 何 凤, 杨 兵, 张厚玉, 刘随军, 谷 新, 刘丹丹, 刘晓冬, 于景生, 马於光. 物理化学学报, 2009, 25: 869]

20 Yang, B.; Liu, X. D.; Xu, H.; Zheng, Y.; Lu, P.; Yu, J. S.; Ma, Y. G.; Feng, J. K. Acta Phys. -Chim. Sin., 2006, 22: 962 [杨 兵, 刘晓冬, 许 海, 郑 岩, 路 萍, 于景生, 马於光, 封继康. 物理 化学学报, 2006, 22: 962]

21 Wang, J. F.; Feng, J. K.; Ren, A. M.; Liu, X. D.; Ma, Y. G.; Lu, P.; Zhang, H. X. Macromolecules, 2004, 37: 3451

22 Yu, J. S. K.; Chen, W. C.; Yu, C. H. J. Phys. Chem. A, 2003, 107:
4268

23 Marcus, R. A. J. Chem. Phys., 1965, 43: 679

24 Marcus, R. A. Rev. Mod. Phys., 1993, 65: 599

25 Yang, X. D.; Li, Q. K.; Shuai, Z. G. Nanotechnology, 2007, 18: 424029

26 Yin, S. W.; Yi, Y. P.; Li, Q. K. J. Phys. Chem. A, 2006, 110: 7138

27 Valeev, E. F.; Coropceanu, V.; da Silva Filho, D. A. J. Am. Chem. Soc., 2006, 128: 9882

28 Song, Y. B.; Di, C. A.; Yang, X. D. J. Am. Chem. Soc., 2006, 128: 15940

29 Yang, B.; Kim, S. K.; Xu, H.; Park, Y. I.; Zhang, H. Y.; Gu, C.; Shen, F. Z.; Wang, C. L.; Liu, D. D.; Liu, X. D.; Hanif, M.; Tang, S.; Li, W. J.; Li, F.; Shen, J. C.; Park, J. W.; Ma, Y. G. ChemPhysChem, 2008, 9: 2601

30 Malagoli, M.; Brédas, J. L. Chem. Phys. Lett., 2000, 327: 13

31 Li, M.; Lu, P.; Zhang, H. Q.; Lu, D.; Ma, Y. G. Chinese Journal of Luminescence, 2006, 27(1): 80 [李 茂, 路 萍, 张海全, 陆 丹, 马於光. 发光学报, 2006, 27(1): 80 ]

32 Lu, H. F.; Chan, H. S. O.; Ng, S. C. Macromolecules, 2003, 36 1543

$33 \mathrm{Lu}, \mathrm{P}$. The synthesis, electronic structure and optoelectronic properties of some polyfluorene derivatives [D]. Jilin: Jilin University, 2005 [路 萍. 若干聚芴衍生物的合成、电子结构与 光电性质的研究[D]. 吉林: 吉林大学, 2005]

34 Lukes, V.; Aquino, A.; Lischka, H. J. Phys. Chem. A, 2005, 109 10232

35 Ma, J.; Li, S. H.; Jiang, Y. S. Macromolecules, 2002, 35: 1109

36 Hsu, C.; Hirata, S.; Head-Gordon, M. J. Phys. Chem. A, 2001, 105: 451

37 Gierschner, J.; Cornil, J.; Egelhaaf, H. J. Adv. Mater., 2007, 19: 173 